\title{
Magnetization of carbon-coated ferromagnetic nanoclusters determined by electron holography
}

\author{
S. Seraphin ${ }^{\text {a) }}$ \\ Department of Materials Science and Engineering, University of Arizona, Tucson, Arizona 85721 \\ C. Beeli ${ }^{\mathrm{b})}$ \\ Centre Interdépartemental de Microscopie Electronique, Ecole Polytechnique Fédérale de Lausanne, \\ CH-1015 Lausanne, Switzerland \\ J-M. Bonard \\ Institut de Physique Expérimentale, Ecole Polytechnique Fédérale de Lausanne, \\ CH-1015 Lausanne, Switzerland \\ J. $\mathrm{Jiao}^{\mathrm{c})}$ \\ Department of Materials Science and Engineering, University of Arizona, Tucson, Arizona 85721 \\ P.A. Stadelmann \\ Centre Interdépartemental de Microscopie Electronique, Ecole Polytechnique Fédérale de Lausanne, \\ CH-1015 Lausanne, Switzerland
}

A. Châtelain

Institut de Physique Expérimentale, Ecole Polytechnique Fédérale de Lausanne, CH-1015 Lausanne, Switzerland

(Received 30 November 1998; accepted 2 April 1999)

\begin{abstract}
The magnetic properties of carbon-coated $\mathrm{Co}$ and Ni nanoparticles aligned in chains were determined using transmission electron holography. The measurements of the phase change of the electron wave due to the magnetization of the sample were performed. The ratio of remnant magnetization to bulk saturation magnetization $M_{\mathrm{r}} / M_{\mathrm{s}}$ of Co decreased from 53\% to $16 \%$ and of Ni decreased from $70 \%$ to $30 \%$ as the particle diameter increased from 25 to $90 \mathrm{~nm}$. It was evident that the inhomogenous magnetic configurations could diminish the stray field of the particles. After being exposed to a 2-Tesla external magnetic field, the $M_{\mathrm{r}} / M_{\mathrm{s}}$ of Co increased by $45 \%$ from the original values with the same dependency on the particle size. The $M_{\mathrm{r}} / M_{\mathrm{s}}$ of Ni particles, on the other hand, increased only $10 \%$. The increased magnetization could be attributed to the merging of small domains into larger ones after the exposure to the external magnetic field. The validity of the interpretation of the holograms was established by simulation.
\end{abstract}

\section{INTRODUCTION}

Ferromagnetism is a collective phenomenon which is caused by the mutual interaction of many atoms and their electrons. As a consequence, the size, composition, microstructure, and morphology of a ferromagnetic sample influence its magnetic properties. Various models have been used to interpret different aspects of ferromagnetism. ${ }^{1}$ The interaction can be considered limited to nearest neighbors, placing emphasis on the properties of

\footnotetext{
a) Address all correspondence to this author. e-mail: seraphin@u.arizona.edu

${ }^{b)}$ Present address: Laboratory of Solid State Physics, ETH Zurich, CH-8093 Zurich, Switzerland.

${ }^{c}$ Present address: Department of Physics, Portland State University, Portland, OR 97207, U.S.A.
}

the atoms involved (Heisenberg model). Other models require that the itinerant properties of the electrons are considered (bandstructure model). The reality lies in between both models, dependent on the size and microstructure of the sample.

The understanding gained new inputs by the possibility to prepare small clusters of ferromagnetic materials with less than a thousand atoms and dimensions approaching the nanometer. ${ }^{2}$ The number of atoms contained in these clusters is small enough to consider only a fraction of them belonging to the bulklike core while the sizable remainder is affected by their closeness to the surface. It can be expected that one or the other ferromagnetic mechanism is predominantly present in either the core or the surface region. If the fractional contribution of surface and bulk varies, the corresponding magnetic behavior of the clusters will change. Therefore, 
their magnetic properties will vary with the diameter of the particles and the arrangement of atoms as determined by the crystal structure. Surface features such as oxidation or encapsulation will also change the fractional contribution of the bulk versus surface regions.

The field gained additional input by the discovery of methods to encapsulate ferromagnetic metals and their alloys into cages of multilayer graphite. ${ }^{3-8}$ The interest in these encaged nanoscale magnets was stimulated by their technological promise. Recording technologies in particular are searching for very fine-grained magnetic materials that could be tailored in their magnetic properties while being resistant to oxidation. It was shown that the graphite cages can protect the ferromagnetic cores against oxidation during longterm immersion in acids. ${ }^{4,11}$ A second, more fundamental reason was that the limited size and number of atoms in a particle could support novel physical properties that were not stable in the macroscopic bulk. Indeed, crystalline structures of the metal encapsulates were observed in temperature regions that were not representative of the bulk-phase diagram. For example, carbon-coated Co nanoparticles were found to have face-centered-cubic (fcc). phase instead of hexagonalclose-packed (hcp) which is usually stable in the bulk at room temperature. ${ }^{4}$ The presence and the effect of the tightly confining graphite cage could transform inner carbon shells into diamond, ${ }^{9}$ to give another particularly striking example.

It is possible to control the diameter of carbon-coated nanoparticles of ferromagnetic elements by varying the preparation conditions such as the size of the anodic metal pool, the jet flow rate, and the static pressure in the carbon-arc-based method of preparation. ${ }^{10,11}$ This raised the question of how their magnetic properties were affected by their size or encapsulation. Several studies were devoted to answering this question. Samples of different composition, structure, and morphology were prepared, their sizes and composition characterized by electron microscopy and spectroscopy, and their integral magnetic properties measured, as a function of temperature. ${ }^{4,7,11,12}$ It was confirmed that the encapsulated particles were indeed ferromagnets which makes them interesting from a technological point of view. Although several explanations could be cited for the interpretation of the results, a decision among them was complicated by a lack of information on the magnetic properties of individual particles because only integral magnetic measurements were performed up to now on the samples. The only report on the magnetic characterization of individual carbon-coated nanoparticles $(\mathrm{Co}, \mathrm{Ni}$, Dy) was focused on ellipsoidal particles of 15-30 nm long diameter. ${ }^{13}$ The results were obtained by superconducting quantum interference magnetometry specially designed for micrometer-size characterization and showed that the nanoparticles are of a single-domain character.
A recently developed technique for measuring magnetization of the particles of small dimensions is electron holography. The technique allows the measurement of the phase change of an electron wave due to the magnetization of the sample. ${ }^{14,15}$ Thin nanowires of ferromagnetic materials have been characterized using electron holography with respect to their domain structure as a function of diameter and length-to-diameter ratio. ${ }^{16,17}$ The study presented here uses electron holography to determine the magnetization of spherical carbon-coated $\mathrm{Co}$ and Ni particles as a function of their diameters (25 to $90 \mathrm{~nm}$ ). The principles and practical aspects of the electron holography are presented in the following sections. The limitations of resolution are discussed and simulations are presented that support the statements made concerning the magnetization of the individual particles.

\section{EXPERIMENTAL PROCEDURES}

\section{A. Preparation of carbon-coated cobalt and nickel particles}

The carbon-coated nanoparticles were prepared in a modified arc discharge chamber ${ }^{4}$ with two vertical electrodes facing each other. A graphite rod of $6.5 \mathrm{~mm}$ diameter was located on the top as the cathode. The anode was a graphite crucible with an inner diameter of $25 \mathrm{~mm}$, filled with either cobalt or nickel. A jet of helium gas at a velocity of approximately $30 \mathrm{~m} / \mathrm{s}$ was introduced in the direction perpendicular to the electrodes. The arc discharge was set at $22 \mathrm{~V}, 175 \mathrm{~A}$ in dc current under a helium pressure of 300 Torr. The deposit was collected and examined in scanning and transmission electron microscopes (SEM/TEM). It consisted of only spherical carbon-coated metal particles without any tubular or other unwanted structures [Fig. 1(a)]. A typical TEM image shown in Fig. 1(b) reveals that most of the particles are coated with a thin graphite layer (inset). Our samples consisted of a mixture of completely coated particles and a fraction of incompletely coated particles.

To prepare samples for electron holography, the deposit was dispersed in ethanol and sonicated for $5 \mathrm{~min}$. The suspension was spread on a standard TEM grid which was covered with a continuous thin film of amorphous carbon without holes. Two sets of samples for each metal were studied: as deposited and after magnetization in a 2-Tesla magnetic field applied in a direction parallel to the grid plane. Only the chains of particles that were parallel to the applied magnetic field were investigated in electron holography on the same day for $\mathrm{Ni}$ and 2 days later for Co. About 15 to 20 chains of each sample type were studied.

\section{B. Electron holography}

Electron holography is one of the few techniques available for measuring the microscopic magnetization. 


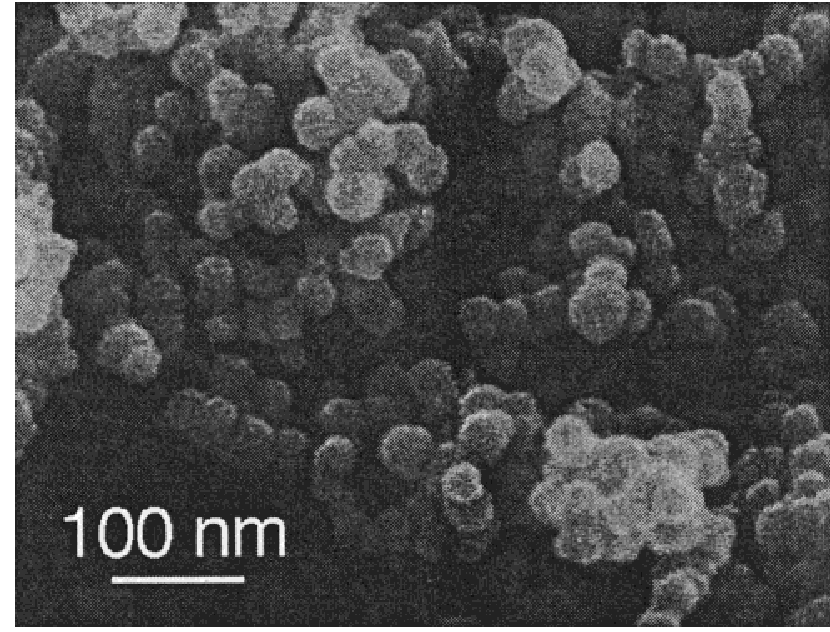

(a)

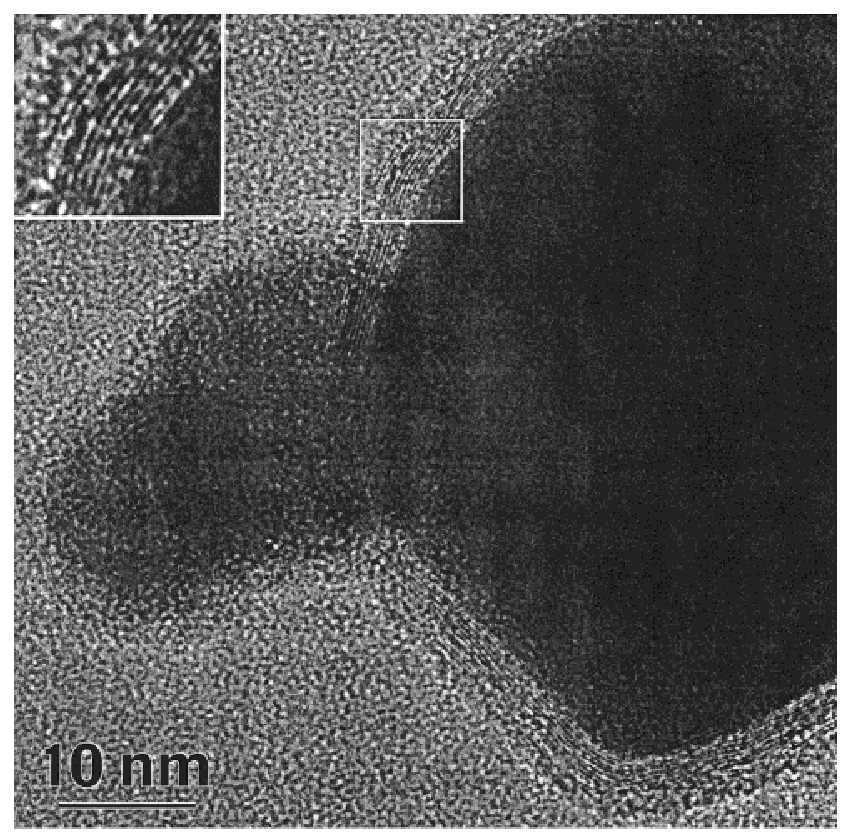

(b)

FIG. 1. (a) SEM image of Ni particles illustrating the size and the shape of the particles. (b) TEM image of Ni particles showing the graphitic coating (inset).

The principle is based on the fact that the electron wave transmitted through a magnetic sample changes phase depending on the sample magnetization and its thickness. For spherical particles, the phase change due to the sample thickness will be symmetric around the particle center, while the phase change due to the magnetization will be asymmetric across the particle. The phase change can be retrieved from a hologram obtained by overlapping the wave scattered by the sample with the (partially) coherent reference wave (Fig. 2). The holograms were reconstructed to retrieve both the amplitude and phase of a wave scattered by the sample. The phase image reconstructed from the hologram corresponds to the phase dif-

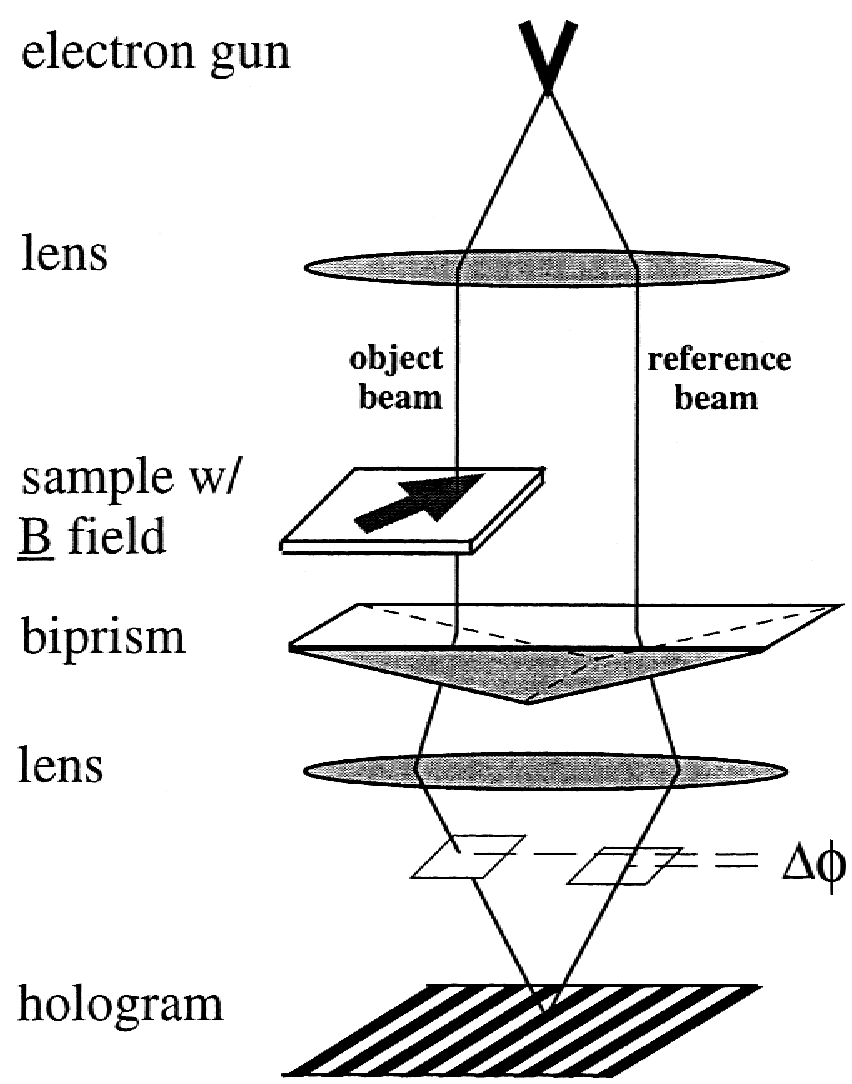

FIG. 2. Schematic drawing of the experimental set up for recording electron holograms of magnetic specimens. For a magnetic sample, the phase difference $\Delta \phi$ is proportional to the magnetic flux density $\mathrm{B}$ integrated over an area limited by a reference beam and an object beam along the direction of the optical axis of the microscope.

ference, $\Delta \phi$, between object and reference waves. For a magnetic field, the phase difference is proportional to the integral of the magnetic-flux density B over an area between reference beam and object beam along the direction of the optical axis of the microscope as displayed in Fig. 2. In determining the magnetic properties of the particles, it will be most useful to obtain the magnetic field lines inside the particles. This requires a TEM with an additional Lorentz lens to provide the hologram resolution sufficient to resolve the radius of the particles. ${ }^{18}$ Alternatively, one can measure the magnetic field outside the particles, i.e., the leakage field. However, the hologram resolution and the phase sensitivity in our set-up (see below) are insufficient to reliably measure the leakage field of a single isolated particle smaller than $50 \mathrm{~nm}$. Therefore, we selected particles aligned in linear chains. The clusters of particles are avoided in our measurement because the magnetization of individual particles will partially cancel each other resulting in a reduction of the leakage field. Furthermore, the inner mean potential of these small particles is negligible in the phase-change retrieval because of the low resolution of our holograms.

For a chain of nanoparticles, the flux of B through the chain itself corresponds to the total flux leaking from the 
chain and can thus be measured as a phase change by electron holography. The phase change $\Delta \phi$ across the chain of the particles is proportional to the total leakage flux of the chain $\left(\mu_{0} M S\right)$. It is given by:

$$
\Delta \phi=(2 \mathrm{e} \pi / \mathrm{h}) \mu_{0} M S,
$$

where $S$ is the cross-sectional area of the particle, $M$ its magnetization, and $\mu_{O}$ is the magnetic field constant $\left(4 \pi \times 10^{-7} \mathrm{Vs} / \mathrm{Am}\right)$. The magnetization $M$ which is the magnetic moment of the remnant state $M_{\mathrm{r}}$ can be determined when the value of $\Delta \phi$ is measured from the reconstructed phase image retrieved from the holograms, while $S$ is calculated from the diameter of the particle measured from a conventional TEM image. We determined $M_{\mathrm{r}}$ for particles of various diameters. To illustrate the dependence of the magnetization on the particle size, the $M_{\mathrm{r}}$ values were normalized with respect to the bulksaturation magnetization, $M_{\mathrm{s}}\left(5.1 \times 10^{5} \mathrm{~A} / \mathrm{m}\right.$ for $\mathrm{Ni}$ and $1.4 \times 10^{6} \mathrm{~A} / \mathrm{m}$ for $\mathrm{Co}$ ). This is acceptable because the saturation magnetization of the particles is not by itself dependent on the size of the particles once they are bigger than $20 \mathrm{~nm} .{ }^{11}$ While the saturation magnetization of the particles rises strongly for diameters between 5 and $20 \mathrm{~nm}$, it levels off at $85 \%$ of the bulk value once the particles have a diameter greater than $20 \mathrm{~nm}$, as is the case in this study.

It should be pointed out that electron holography can detect only the in-plane component of magnetization along the chain and not the vertical component. However, the fact that the particles arrange themselves in a linear chain indicates that the direction of the magnetization is along the chain and in the plane of measurement. Furthermore, this procedure usually gives magnetization values too small if there is partial flux closure inside the particles as in the case of multiple domain particles. On the other hand, it will give too large a value for small particles (smaller than $50 \mathrm{~nm}$ ) that are attached between two larger particles. The measurement will be more reliable if the adjacent particles have similar size which is usually the case in our measurement.

The experimental micrographs were obtained using a Hitachi HF-2000 FEG equipped with a rotatable electrostatic biprism. A negative voltage $(-7$ to $-9 \mathrm{~V})$ was applied to the biprism fiber to superimpose the reference wave and the object wave. The width of the interference region depended on the voltage applied to the biprism fiber and was typically 2 to $3 \mathrm{~mm}$. The holograms were recorded using a Gatan retractable slow-scan CCD camera. To study the remnant magnetization state of the sample, the objective lens current was switched off. The specimen had to be imaged with the first intermediate lens while the remaining three lenses were fully excited to reach the maximum possible magnification in this configuration (1100 times on the CCD camera at an operat- ing voltage of $200 \mathrm{kV}$ ). To further magnify the final image, the operating voltage of the microscope was reduced to $100 \mathrm{kV}$ because the given lens strength is more effective on the slower electrons. The magnification amounted to 2800 times on the CCD camera. ${ }^{16,17}$ The remnant magnetization of the particles was investigated by TEM electron holography at $100 \mathrm{kV}$ while their diameters were measured by conventional TEM at $200 \mathrm{kV}$. In our case, the hologram spatial resolution was around $100 \mathrm{~nm}$ (which corresponds to 2 or 3 times the interference fringe spacing) and the phase resolution was \pm 0.2 radian. To demonstrate that the obtainable resolution is sufficient to determine the magnetization of the carboncoated nanoparticles of 25 to $90 \mathrm{~nm}$, a series of simulations was performed. The interpretation of the phase maps is supported by these simulations presented below.

\section{RESULTS AND DISCUSSION}

\section{A. Magnetization of carbon-coated $\mathrm{Ni}$ and Co nanoparticles}

In each type of $\mathrm{Ni}$ or Co sample, 15 to 20 holograms were taken. Each phase image reconstructed from a hologram provided 8 to 12 values of the phase change depending on the shape of the chains and the quality of holograms. Overall, about 120 particles in each sample were analyzed. The particles were classified with respect to their diameters in sections of $5 \mathrm{~nm}$ width centered at $25,30, \ldots, 60 \mathrm{~nm}$ for Co case, and the mean magnetization of each diameter range was reported. For Ni particles, sections of $10 \mathrm{~nm}$ width centered at $35,45, \ldots$, $85 \mathrm{~nm}$ were used.

Figure 3(a) shows an image of a Ni chain; Fig. 3(b) the reconstructed phase image with three positions labeled 1 , 2 , and 3 where the phase change was measured and plotted as shown in Fig. 3(c). The phase changes across the chain were measured to be $-2.1,-1.6$, and 3.9 radian at location 1, 2, and 3, respectively. At 1, the value of $S$ in Eq. (1) was determined using a particle diameter of $95 \mathrm{~nm}$. From Eq. (1) with $\Delta \phi=-2.1 \mathrm{rad}, M$ was calculated to be $1.5 \times 10^{5} \mathrm{~A} / \mathrm{m}$ which is about $30 \%$ of the bulk saturation magnetization of $\mathrm{Ni}$. The same calculation shows that at location 2 , the phase change of $-1.6 \mathrm{rad}$ across a $70 \mathrm{~nm}$ particle corresponds to $2.2 \times 10^{5} \mathrm{~A} / \mathrm{m}$ (43\% of the bulk saturation magnetization) and at location 3, the phase change of +3.9 rad across a $95-\mathrm{nm}$ particle has $2.9 \times 10^{5} \mathrm{~A} / \mathrm{m}$ ( $57 \%$ of the bulk saturation magnetization). Note that the phase changes the sign from negative values at locations 1 and 2 to a positive value at location 3. It indicates a reversal of magnetic moment along the chain. Fig. 4 shows another example of the sign reversal [indicated by arrows in Fig. 4(a)] along a chain of Co particles. The direction of these magnetic moments along the chain was found to change 
mostly at locations where particles agglomerate together. Figures 4(b) and 4(c) are the amplitude image and the phase image reconstructed from a hologram. Figure 4(d) displays four phase-profiles across the chain and three reversals of magnetization. Note that the spatial resolution of the hologram can be estimated by comparison of the amplitude image, Fig. 4(b) with the TEM image, Fig. 4(a).
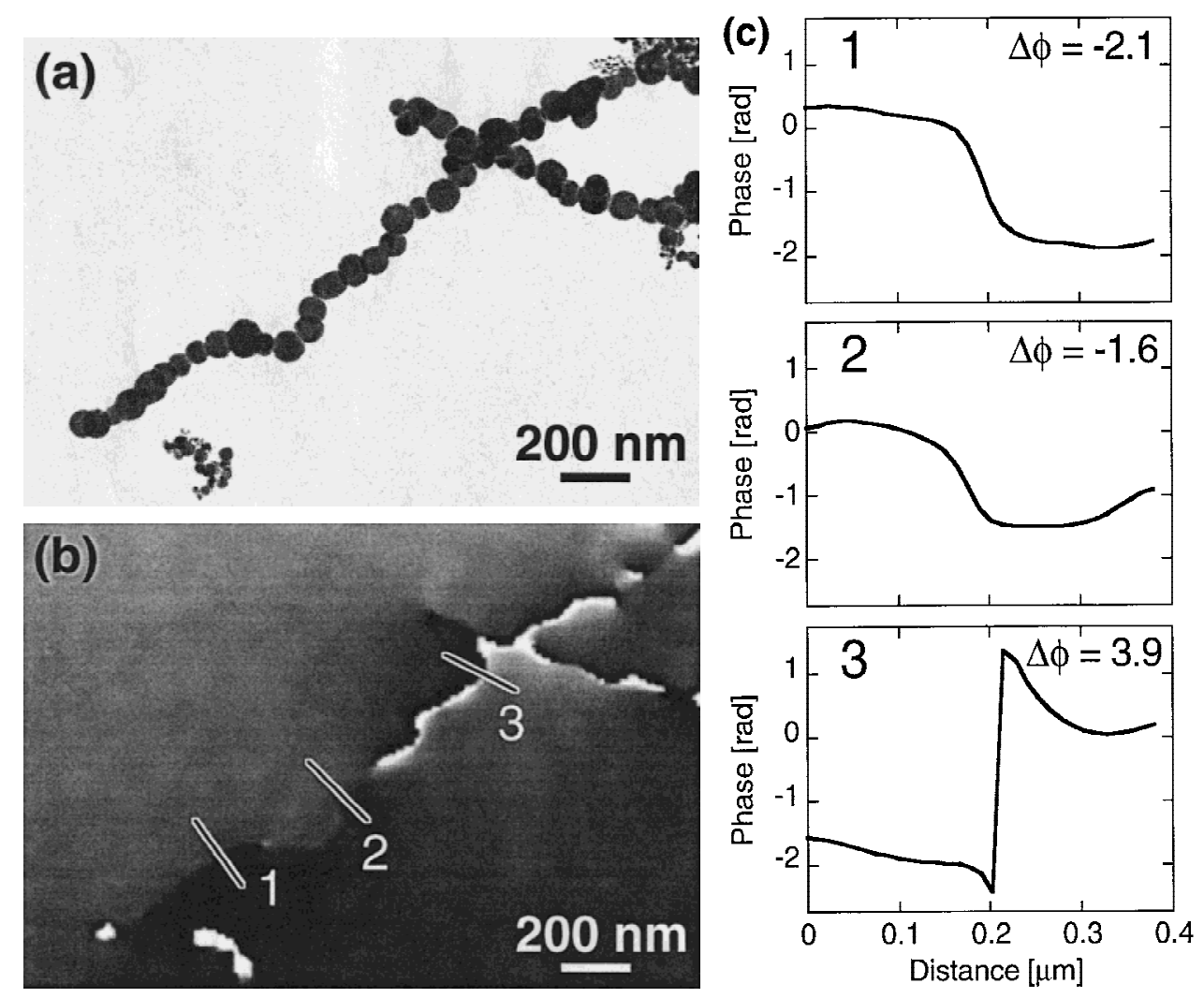

FIG. 3. (a) Image of Ni nanoparticle chains used for holography. (b) Phase map with 1, 2, and 3 indicating the lines along which the phase was plotted and displayed in (c).
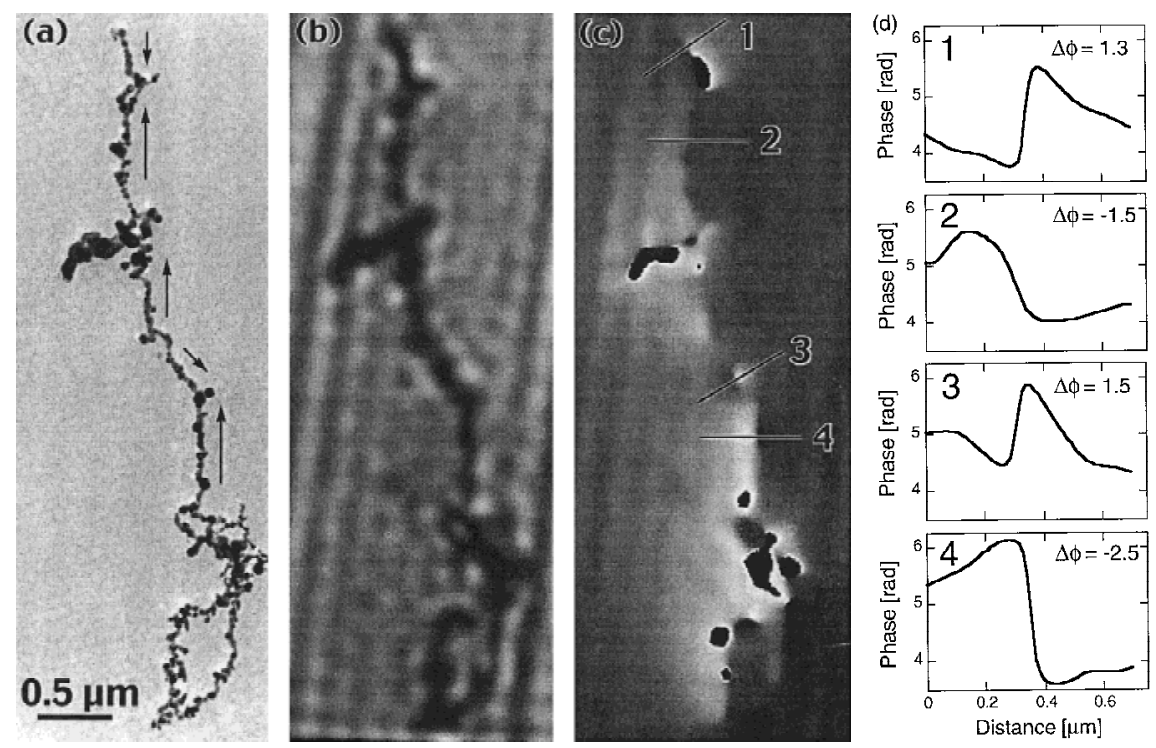

FIG. 4. (a) Chain of Co particles exhibiting reversals of the magnetization along the chain. Arrows indicate the direction of magnetization. (b) Amplitude image. (c) Phase image. The reversal of the magnetization is derived from the sign reversal of the phase change across the chain displayed by profiles in (d). 
Figure 5 shows the relative magnetization of carboncoated Ni nanoparticles as a function of their diameters. The plot reveals that in the diameter range of 30 to $90 \mathrm{~nm}$ the larger the particles the lower the magnetization. The magnetization of the small particles (30-40 nm diameter) is $75 \% \pm 10 \%$, dropping to $32 \% \pm 2 \%$ for particles of 80 to $90-\mathrm{nm}$ diameter. Compared to the integral value of $30 \%$ obtained from the macroscopic measurement of magnetization by VSM technique, ${ }^{4}$ the results from electron holography provide more detailed information on the magnetization of the particles. After exposure to the 2-Tesla external magnetic field, the magnetization of the particles increased about $10 \%$ with the same dependency on the particle size. It can be reasoned that the external field induced the small domains to merge into larger ones, so the internal flux closure is reduced and the leakage of magnetic flux is increased.

Magnetization of carbon-coated Co nanoparticles shows the same trend as $\mathrm{Ni}$ : the larger the particles the lower the magnetization (Fig. 6). However, the ratio of the remnant to bulk saturation magnetization of asdeposited Co is lower than that of Ni. The highest relative magnetization obtained in this sample is around $55 \% \pm$ $6 \%$ and the lowest magnetization is $16 \% \pm 6 \%$. (It should be noted that the bulk saturation magnetization of $\mathrm{Co}$ is $1.4 \times 10^{6} \mathrm{~A} / \mathrm{m}$ and of $\mathrm{Ni}$ is $5.1 \times 10^{5} \mathrm{~A} / \mathrm{m}$ ). This rather low magnetization of Co particles may be due to the presence of multiple domains even in the smallest particles observed, a carbon coating layer and the fcc phase in which cobalt is present here. The fcc is about $5 \%$ inferior in its magnetic property at room temperature to the bulk hcp phase in Co. The decrease of magnetization in larger particles may be due to the flux closure between domains inside the bigger particles. Consequently, the outside magnetic leakage is reduced. Large particles may

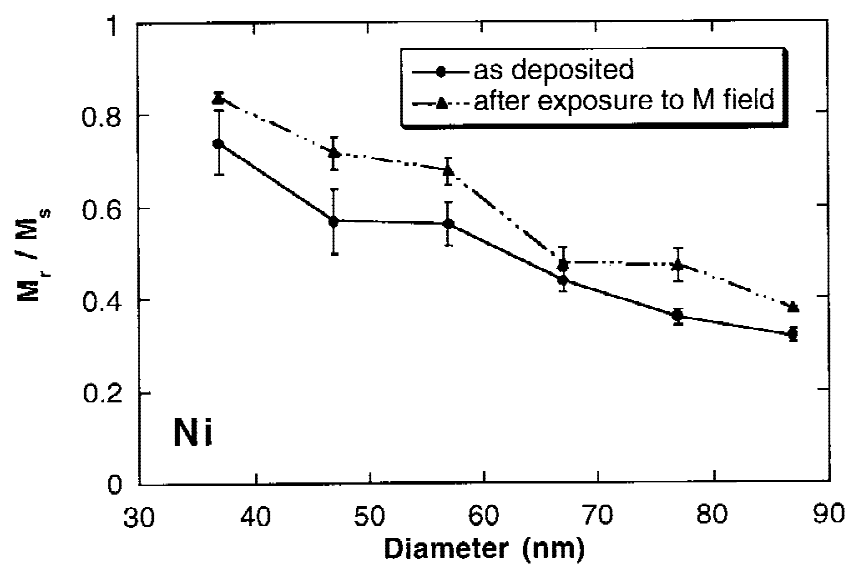

FIG. 5. Relative magnetization $M_{\mathrm{r}} / M_{\mathrm{s}}$ of carbon-coated Ni particles as a function of particle diameter using $M_{\mathrm{s}}=5.1 \times 10^{5} \mathrm{~A} / \mathrm{m}$. Exposure to an external magnetic field ( 2 Tesla) increases the magnetization of the particles by approximately $10 \%$.

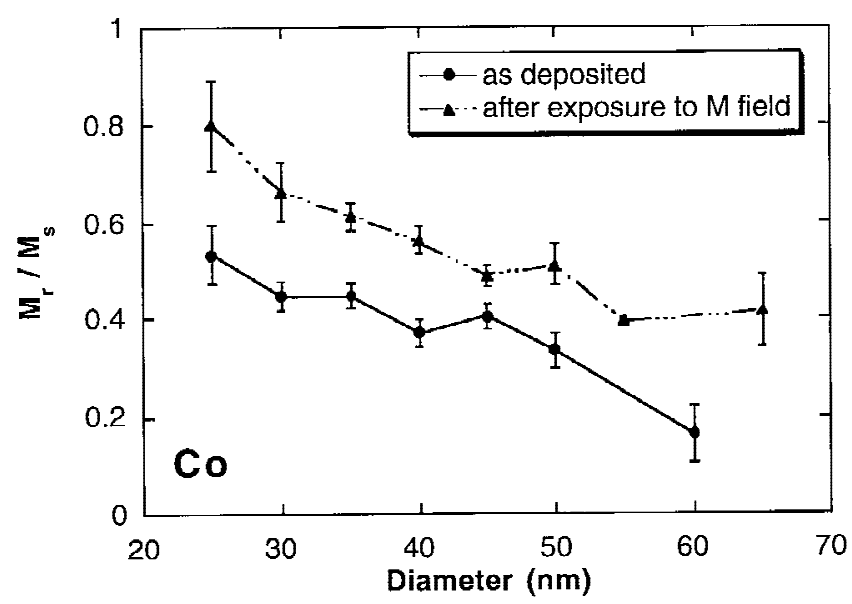

FIG. 6. Relative magnetization $M_{\mathrm{r}} / M_{\mathrm{s}}$ of carbon-coated Co particles as a function of particle diameter using $M_{\mathrm{s}}=1.4 \times 10^{6} \mathrm{~A} / \mathrm{m}$. Exposure to an external magnetic field ( 2 Tesla) increases the magnetization of the particles by approximately $45 \%$.

also have their individual magnetic moments out of the plane and not along the chain direction. This effect will reduce the detectable phase shift in the hologram because the electron holography technique can determine only the in-plane component of the magnetic flux. After being exposed to the 2-Tesla external magnetic field, the magnetization of Co particles increases $45 \%$ compared to the as-deposited particles with a similar dependence on particle size. The larger increase (45\%) of the magnetization after exposure to the external field in Co compared to only $10 \%$ in $\mathrm{Ni}$ can be related to an observed higher defect density in Co particles. Because Co particles are fcc, most defects are likely to be stacking faults which results in a locally hcp phase (for example, intrinsic stacking fault $\mathrm{ABCABABCABC}$ or extrinsic stacking

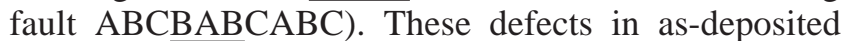
particles cause pinning of opposite magnetic spins resulting in multiple domain particles. Obviously, the 2-Tesla external field was strong enough to align the magnetic spin across the defects and reduced the domain boundaries. This effect lasted at least 2 days judging from the time of the exposure to the time of the holography analysis.

\section{B. Interpretation of phase maps}

To understand the influence of the limited spatial resolution on the reconstructed phase maps, a series of simulations has been performed. They address basically three different aspects of the measurements. First, we show that the evaluation of the magnetization from an external leakage field is meaningful to the extent that the magnetization can be expressed in the form of the dipole arrows shown in Fig. 4(a), independent of the size of the particles and the possible presence of multiple magnetic 
domains inside the particle. Second, the inversion of the direction of magnetization is directly observable. Third, the limited resolution of the holograms does not interfere with an accurate statement about the phase shift due to the magnetic flux. The following simulations will substantiate all three claims. The simulations were carried out by Mathematica and HoloWorks ${ }^{19}$ software. Mathematica was used to stimulate the phase maps which were subsequently used to calculate with HoloWorks a set of holograms with different spatial-resolution values. In the simulation of the electron holograms the influence of a perturbed reference wave due to the far field (leakage field) of the magnetic sample was taken into account. The reconstruction of these holograms will be presented in the following paragraphs.

Real magnetic (monodomain) particles have a far field similar to a dipole field, however, the field variations near the particle or across the particle differ significantly from a dipole field, i.e., it does not have the mathematical discontinuity across the dipole line. Figure 7(a) presents the ideal phase map of a small cylindrical particle with $150 \mathrm{~nm}$ in length and $60 \mathrm{~nm}$ in diameter, which is comparable to a segment of our nanoparticle chains. A magnetization corresponding to $1.3 \times 10^{6} \mathrm{~A} / \mathrm{m}$ (approximately the bulk value of cobalt) has been assumed. The corresponding phase profile across the particle is shown in Fig. 7(b). The phase varies continuously across the particle without any abrupt phase changes and reveals a total phase shift of $7 \mathrm{rad}$. A simulated hologram with high resolution [Fig. 7(c)], i.e., with fine interference fringes of $9 \mathrm{~nm}$ distance, provides a reconstructed phase map which correctly represents the continuous-phase change across the particle. Because the total phase change across the particle exceeds $2 \pi$, the phase is "wrapped" in the reconstructed phase map [Fig. 7(d)], which represents the phase in the interval from $-\pi$ to $\pi$. The closed discontinuity line which bounds the kidney-shaped, black region in Fig. 7(d) corresponds to a $2 \pi$-discontinuity line. This phase map, therefore, can easily be "unwrapped" by adding $2 \pi$ to all the data points inside the black region bounded by the discontinuity line to reconstruct the physical-phase information. The phase profile [Fig. 7(e)] across the particle shows a $-2 \pi$ and $a+2 \pi$ phase discontinuity when crossing the $2 \pi$ discontinuity line. Nevertheless, the close correspondence to the original-phase profile in Fig. 7(b) can be recognized. By adding $2 \pi$ to the part between the two $2 \pi$-phase discontinuities, a total phase change of $6.8 \pm 0.1 \mathrm{rad}$ can be measured from this profile.

In contrast, the hologram presented in Fig. 7(f) has low spatial resolution (interference fringe spacing of $34 \mathrm{~nm}$ ). The interference fringes running across the particle are strongly bent, because the magnetic flux enclosed between two adjacent fringes is much larger than in the case of the fine interference fringes of Fig. $7(\mathrm{c})$. The $2 \pi$ dis- continuity line in the corresponding reconstructed phase map [Fig. 7(g)] is not a closed loop and thus, does not allow a correct unwrapping of the phase information (i.e., without the help of further knowledge on the physical nature of the specimen). Nevertheless, the total phase change across the particle can be measured with a phase profile [Fig. 7(h)]. The measured value of $6.8 \pm 0.2 \mathrm{rad}$ is reasonably close to the value obtained in Fig. 7(e) and compares well with the original value. However, the measurement uncertainty is slightly larger than in the case of the profile in Fig. 7(e).

These examples have shown that a low spatial resolution of the electron hologram leads to phase maps with certain artifacts, especially if the phase changes more than $2 \pi$ over a short distance. The measurement of the total phase change is nevertheless possible if care is taken to correctly interpret the $2 \pi$ discontinuity. Generally, the phase maps with low spatial resolution lead to a reduced sensitivity of the phase measurement. However, the present simulations indicate that the conditions used in our experiments still allow a reasonable measurement accuracy.

The orientation of the magnetization along the chain of particles has been found to change direction (shown in Figs. 3 and 4). In the following simulation, we illustrate that the observation of the reversal of magnetization direction along the chain is straightforward. In Fig. 8(a), two identical magnetic segments with opposite magnetization direction are presented in an ideal phase map. The opposite direction of the magnetization can be recognized by the opposite side of the bright contrast with respect to the magnetic dipoles. In this simulation, each magnetic segment is $150 \mathrm{~nm}$ long, $60 \mathrm{~nm}$ in diameter, and the magnetization is $5.5 \times 10^{5} \mathrm{~A} / \mathrm{m}$ (slightly larger than the bulk value for nickel). This results in a total phase change of $3 \mathrm{rad}$ across the magnetic segment. The corresponding hologram [Fig. 8(b)] has been simulated with an interference fringe spacing of $34 \mathrm{~nm}$. The interference lines are shifted in opposite directions because of the opposite magnetization direction of the segments. Because the shift can be very weak in experimental holograms this cannot be observed as easily as in the presented simulations. The reversal of the magnetization direction is, however, revealed in the reconstructed phase map [Fig. 8(c)], because the basic pattern of Fig. 8(a) is clearly recovered. The phase profiles across the two segments are presented in Figs. 8(d) and 8(e), respectively. While the phase profile in Fig. 8(d) displays a phase change from approximately +1.5 to $-1.5 \mathrm{rad}$, the phase profile in Fig. 8(e) gives the opposite phase change. This is another clear indication that the direction of the magnetization is opposite for these two segments. It is shown that the reversal of magnetization is an obvious observation against any possible artifact caused by the experimental set-up or conditions. 

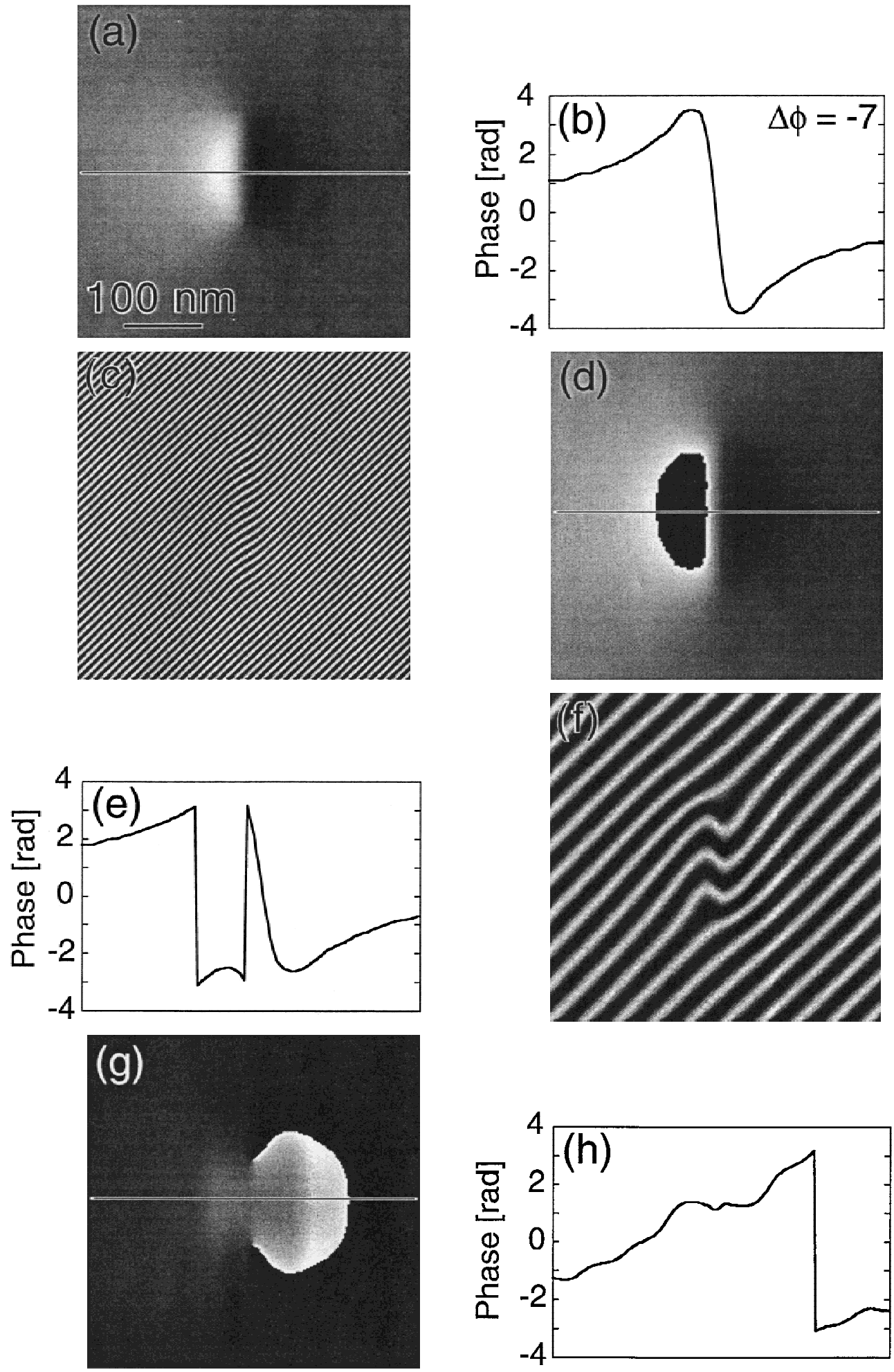

FIG. 7. (a) Ideal phase map of a small cylindrical particle with $150 \mathrm{~nm}$ in length, $60 \mathrm{~nm}$ in diameter, and a magnetization of $1.3 \times 10^{6} \mathrm{~A} / \mathrm{m}$. (b) The phase profile across the particle showing the continuous phase change with a total shift of 7 rad. (c) Simulated hologram with high spatial resolution of $9 \mathrm{~nm}$ interference fringe spacing. (d) Reconstructed phase map showing a $2 \pi$ discontinuity line surrounding the kidney-shaped black region. (e) Phase profile across the particle. By adding $2 \pi$ to the part between the two $2 \pi$ phase discontinuities a total phase change of $6.8 \pm 0.1$ rad can be measured. (f) Simulated hologram with low resolution of $35 \mathrm{~nm}$ interference fringe spacing. (g) Reconstructed phase map showing a $2 \pi$ discontinuity line that is not a closed loop. (h) Phase profile across the particle revealing a total phase shift of $6.8 \pm 0.2 \mathrm{rad}$. All figures have the same scale as Fig. 7(a). 

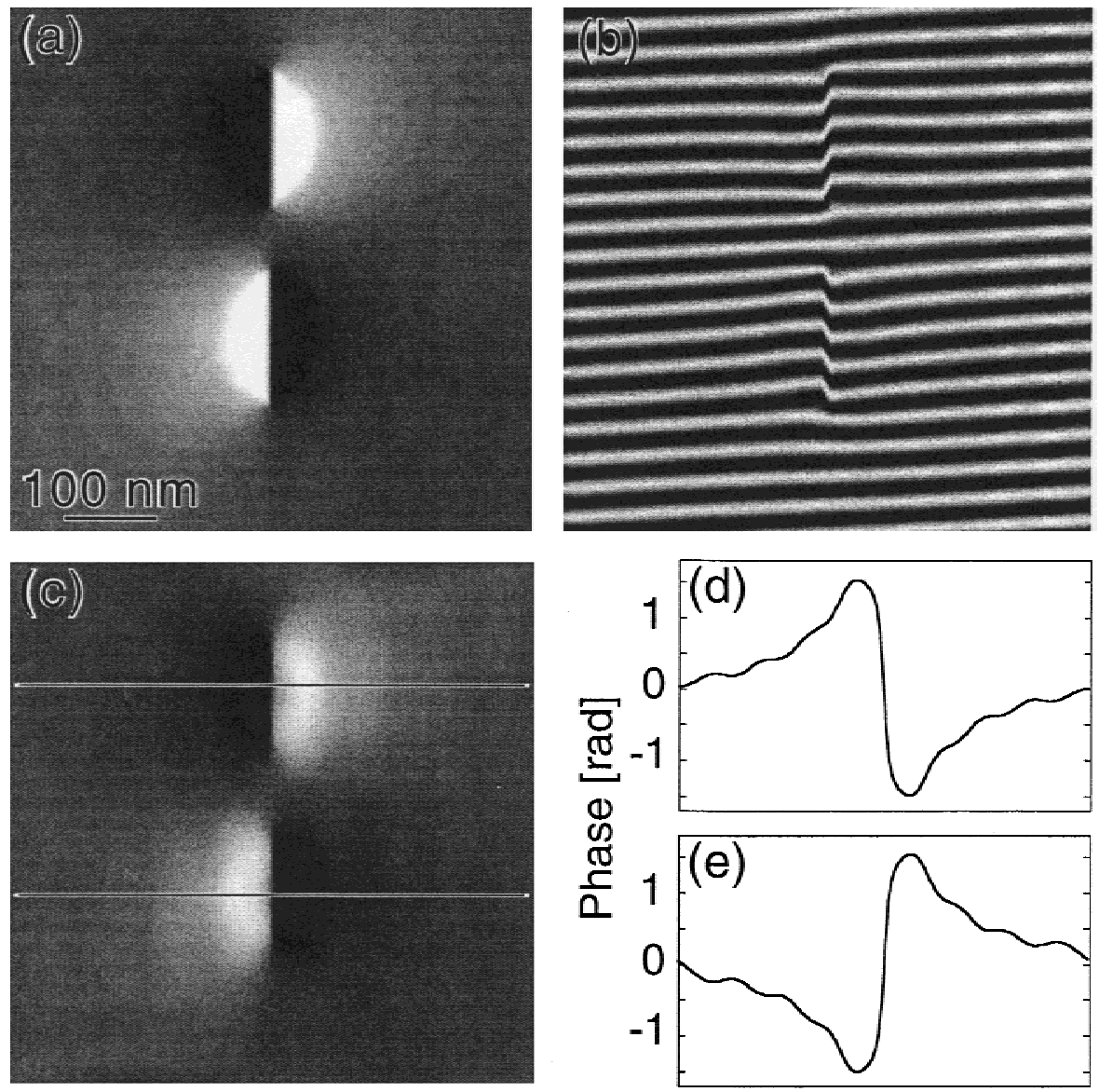

FIG. 8. (a) Ideal phase map of two identical magnetic segments with opposite magnetization direction. Each magnetic segment is $150 \mathrm{~nm}$ long, $60 \mathrm{~nm}$ in diameter, and the magnetization is $5.5 \times 10^{5} \mathrm{~A} / \mathrm{m}$. (b) Simulated hologram with an interference fringe spacing of $34 \mathrm{~nm}$. The interference lines are shifted in opposite directions because of the opposite magnetization direction of the segments. (c) Reconstructed phase map. (d) and (e) The phase profiles across the two segments. While the phase profile in Fig. 8(d) displays a phase change of -3 rad, the phase profile in Fig. 8(e) gives the opposite phase change of $+3 \mathrm{rad}$.

\section{CONCLUSIONS}

The observations reported above lead to the following conclusions:

(1) Electron holography by transmissions electron microscopy can be used to determine the remnant magnetization of individual carbon-coated nanoparticles of $\mathrm{Co}$ and $\mathrm{Ni}$ attached to each other in chains. The magnetization of the sample is observed through the phase change of the electron wave transmitted through the sample. Simulations of the phase maps show that the conditions used in the experiments allow a reasonable measurement accuracy.

(2) The direction of magnetization is usually along the chain. Changes in the magnetic direction can be derived by the reversal of a positive to a negative change of the electron-wave phase. Polarization of the particle chains caused by exposure to a strong external magnetic field results in an increase of the observed remnant magneti- zation. It is speculated that the increased magnetization is due to the merging of domains into larger ones. Differences between $\mathrm{Co}$ and $\mathrm{Ni}$ can be explained by the greater tendency of Co nanoparticles to contain stacking faults.

(3) Above a particle diameter of $25 \mathrm{~nm}$, the relative magnetization decreases from $53 \%$ to $16 \%$ of the bulk value in Co as the size of the particle increases to $70 \mathrm{~nm}$. Corresponding values for $\mathrm{Ni}$ are $70 \%$ and $30 \%$ for the diameter ranging from 30 to $90 \mathrm{~nm}$. The tendency to form multidomain structures resulting in flux closure inside the bigger particles may provide an explanation.

\section{ACKNOWLEDGMENTS}

We acknowledge the financial support of the U.S. National Science Foundation (Grant No. DMR 9302353), the Swiss National Science Foundation, and the Ecole Polytechnique Fédérale de Lausanne, Switzerland. 


\section{REFERENCES}

1. D.C. Mattis, The Theory of Magnetism, 2nd ed. (Springer-Verlag, Berlin, 1988).

2. I.M.L. Billas, A. Châtelain, and W.A. de Heer, Science 265, 1682 (1994).

3. J-H. Hwang, V.P. Dravid, M.H. Teng, J.J. Host, B.R. Elliott, D.L. Johnson, and T.O. Mason, J. Mater. Res. 12, 1076 (1997).

4. J Jiao and S. Seraphin, J. Appl. Phys. 80, 103 (1996).

5. Y. Saito, M. Okuda, T. Yoshikawa, A. Kasuya, and Y. Nishina, J. Phys. Chem. 98, 6696 (1994).

6. S.A. Majetich, J.O. Artman, M.E. McHenry, N.T. Nuhfer, and S.W. Staley, Phys. Rev. B 48, 16845 (1993).

7. J.J. Host, J.A. Block, K. Parvin, V.P. Dravid, J. L. Alpers, T. Sezen, and R. LaDuca, J. Appl. Phys. 83, 793 (1998).

8. K. Lafdi, A. Chin, N. Ali, and J.F. Despres, J. Appl. Phys. 79, 6007 (1996).

9. F. Banhart, J. Appl. Phys. 81, 3440 (1997).

10. J. Jiao and S. Seraphin, J. Appl. Phys. 83, 2442 (1998).
11. J-M. Bonard, S. Seraphin, C. Beeli, J-E. Wegrowe, T. Stöckli, J. Jiao, P.A. Stadelmann, and A. Châtelain Electrochem. Soc. Proc. 193 (San Diego, CA, 1998), pp. 794-807.

12. T. Hayashi, S. Hirono, M. Tomita, and S. Umemura, Nature 381, 772 (1996).

13. W. Wernsdorfer, E. Bonet Orozco, K. Hasselbach, A. Benoit, B. Barbara, N. Demoncy, A. Loiseau, H. Pascard, and D. Mailly, Phys. Rev. Lett. 78, 1791 (1997).

14. A. Tonomura, Electron Holography, Springer Series in Optical Sciences 70, (Springer, Berlin, 1993).

15. A. Tonomura, L.F. Allard, G. Pozzi, D.C. Joy, and Y.A. Ono, Electron Holography (Elsevier, Amsterdam, 1995).

16. C. Beeli, B. Doudin, J-Ph. Ansermet, P.A. Stadelmann, J. Magn. Magn. Mater. 164, 77 (1996).

17. C. Beeli, B. Doudin, J-Ph. Ansermet, P.A. Stadelmann, Ultramicroscopy 67, 143 (1997).

18. R.E. Dunin-Borkowski, M.R. McCartney, B. Kardynal, D.J. Smith, J. Appl. Phys. 84, 374 (1998).

19. E. Völkl and L. Allard, J. Microsc. 180, 39 (1995). 